\title{
Depression and catastrophizing predict suicidal ideation in tertiary care patients with interstitial cystitis/bladder pain syndrome
}

\author{
Dean A. Tripp, PhD; J. Curtis Nickel, MD; ${ }^{1}$ Adrijana Krsmanovic, MSc; ${ }^{-1}$ Michel Pontari, MD; ${ }^{2}$ \\ Robert Moldwin, MD; ${ }^{3}$ Robert Mayer, MD, ${ }^{4}$ Lesley K. Carr, MD; ${ }^{5}$ Claire C. Yang, MD; Jorgen Nordling, MD
}

'Queen's University, Kingston, ON, Canada; ${ }^{2 T e m p l e ~ U n i v e r s i t y, ~ P h i l a d e l p h i a, ~ P A, ~ U n i t e d ~ S t a t e s ; ~}{ }^{3 H o f s t r a}$ University School of Medicine, New Hyde Park, NY, United States; ${ }^{4}$ Asante Physician Partners, Grants Pass, OR, United States; ${ }^{5}$ University of Toronto, Toronto, ON, Canada; ${ }^{U}$ University of Washington, Seattle, WA, United States; ${ }^{7}$ University of Copenhagen, Herlev, Denmark

Cite as: Can Urol Assoc J 2016;10(11-12):383-8. hittp://dx.doi.org/10.5489/cuaj.3892

\section{Abstract}

Introduction: We sought to evaluate psychosocial factors as predictors of suicidal ideation (SI) in a tertiary care outpatient sample of women suffering from interstitial cystitis/bladder pain syndrome (IC/BPS).

Methods: The patients are women managed at tertiary care centres $(n=190)$. Controls were recruited from the community $(n=117)$. Both groups completed questionnaires on demographics, pain (McGill Pain Questionnaire), IC/BPS symptoms, and psychological variables. Univariate and multivariate hierarchical regression modelling was conducted to examine the strength of associations and unique effects of psychosocial variables on patient SI.

Results: Compared to $6 \%$ in healthy controls, $23 \%$ of patients endorsed SI in the past two weeks. Correlations between SI, depression, and catastrophizing across controls and cases show that for controls, $\mathrm{SI}$ is associated with greater pain $(0.31 ; \mathrm{p}<0.01)$ and depression only $(0.59 ; \mathrm{p}<0.01)$. For tertiary care centre cases, $\mathrm{SI}$ is associated with pain $(0.24 ; p<0.01)$, depression $(0.64 ; p<0.01)$, and catastrophizing $(0.35 ; \mathrm{p}<0.01)$. Regression analyses indicated that psychosocial variables accounted for a significant amount of variance over and above IC/BPS symptoms. Catastrophizing (i.e., helplessness) about pain and depression were significant univariate predictors of SI, but only depression predicted SI in multivariable analyses.

Conclusions: Limitations of this study include its cross-sectional design and primarily correlation-based statistics. The present study is the first to implicate multiple psychosocial risk factors over and above IC/BPS-specific symptoms and patient pain experience in $\mathrm{SI}$ in women with IC/BPS. Depression in particular is uniquely important in predicting suicidality. These results support a multidisciplinary, proactive approach to IC/BPS involving not only treatment of disease symptoms, but also early detection/treatment of associated psychosocial problems.

\section{Introduction}

Interstitial cystitis/bladder pain syndrome (IC/BPS) is a chronic urological condition characterized by chronic pain in the pelvic, abdominal, and lower back areas, accompanied by urinary urgency and frequency, ${ }^{1,2}$ with an unknown etiology and no standardized treatment. ${ }^{3}$ Due to its refractory nature, treatment is focused on symptom and pain management, with psychosocial risk factors like depression as a subordinate concern.

Suicide is the grimmest possible outcome associated with depression, and chronic pain patients endorse greater suicidal ideation (SI) and attempt suicide at a four-fold higher risk than the general population. ${ }^{4}$ While chronic pain conditions are in themselves not considered life-threatening, $\mathrm{SI}$ is an indicator for suicide attempts. Indeed, patients with pain report greater $\mathrm{SI}_{1}{ }^{5}$ attempts, ${ }^{6}$ and completions. ${ }^{6,7}$ Only one study has reported a prevalence of SI among patients with IC/ BPS at $11 \%,{ }^{8}$ with marital status and depression associated with SI over and above IC/BPS-related symptoms.

It is noteworthy that Hepner et al did not evaluate new risk factors, such as catastrophizing, and they employed a community-based epidemiological sample of women who met criteria for RAND interstitial cystitis epidemiology (RICE) high-specificity case definition of IC/BPS, ${ }^{9}$ not a clinical sample with a physician-confirmed diagnosis. Alarmingly, $90 \%$ of unplanned and $60 \%$ of planned first suicide attempts occur within one year of the onset of $\mathrm{SI}_{1}{ }^{10}$ tertiary care patients show greater SI than primary care cases with abdominal-pelvic pathology, ${ }^{11}$ and IC/BPS patients report more depression than controls. ${ }^{12}$ As well, catastrophizing is a predictor of $\mathrm{SI} .{ }^{13}$

This study examined an IC/BPS tertiary care sample and controls for $\mathrm{SI}$ and its biopsychosocial risk factors (i.e., depression, catastrophizing) while controlling for other medical symptoms (pain, urinary symptoms) and demographics. This study adds three novel aims to the urology literature: 1) to be the first estimate of SI point prevalence in 
Tripp et al.

a tertiary care sample; 2 ) to discover the single contributions of depression and catastrophizing as risk factors for SI; and 3 ) to examine the combined risk factors in a multivariable analysis to predict SI.

\section{Methods}

\section{Participants and recruitment}

Patients $(n=190)$ were recruited from IC/BPS clinical practices at participating centres (Manhattan, NY, U.S., $n=26$, 13.7\%; Rochester, NY, U.S., $\mathrm{n}=18,9.5 \%$; Copenhagen, Denmark, $\mathrm{n}=13,6.8 \%$; Toronto, ON, Canada, $\mathrm{n}=15$, 7.9\%; Knoxville, TN, U.S., $\mathrm{n}=11,5.8 \%$; Philadelphia, PA, U.S., $n=6,3.2 \%$; Kingston, ON, Canada, $n=75,39.5 \%$; Washington, DC, U.S., $\mathrm{n}=16,8.4 \%$; Ahmedabad, India, $\mathrm{n}=10,5.3 \%$ ). All centres obtained Research Ethics Board (REB) approval. All patients were English-speaking females. Patients had chronic pelvic pain longer than six months in duration with pressure or discomfort perceived to be related to the bladder, accompanied by at least one other urinary symptom, such as urgency or frequency, and fulfilled the IC/BPS American Urological Association (AUA) diagnostic criteria. ${ }^{3}$ Female healthy controls $(n=117)$ were recruited from the general population by advertisement. At each of the participating sites, patients with IC/BPS were treated concurrently. Identified patients and controls were contacted by telephone to determine interest in this study, then a package with informed consent and the questionnaires were mailed with an enclosed self-addressed, postage-paid envelope from the contacting research centre. Although this study examined data relevant to $\mathrm{SI}$, this study was designed to examine patient quality of life, as was labelled on the consent form and letter of information. This is important because the information collected in this study is thus not impacted by patient bias in regards to direct reporting on suicidal thoughts. All participants were also provided with resources for any emotional concerns raised during participation, a standard procedure in keeping with the REB policies.

\section{Measures}

\section{Demographics}

Participants completed a demographic questionnaire, which included questions on age, education, ethnicity, relationship status, employment status, and length of IC/BPS diagnosis.

\section{Symptoms}

The O'Leary-Sant Interstitial Cystitis Symptom and Problem Indices (ICSI; ICPI) assessed symptomatology. ${ }^{14}$ These are self-report questionnaires consisting of four items. The ICSI assesses symptom severity; participants answer on a scale from 0 ("not at all") to 5 ("almost always/usually"). Total scores range from 0-20. The ICPI assesses the extent to which symptoms were problematic during the last month; participants rate items on a scale from 0 ("no problem") to 4 ("big problem"), with total scores ranging from 0-16.

\section{Pain}

The Short-Form McGill Pain Questionnaire (SF-MPQ) was used to measure self-reported current pain. ${ }^{15}$ Participants specified their pain intensity by indicating the degree to which each of the 15 descriptors described their pain, on a four-point scale from 0 ("none") to 3 ("severe").

\section{Depression}

The Center for Epidemiologic Studies Depression Scale (CES-D) assessed self-reported depression using 20 items. $^{16}$ Participants indicated how often they have associated with a statement over the past week, on a four-point Likert-type scale ranging from 0 ("rarely or none of the time: less than 1 day") $^{\prime \prime} 3$ ("most or all of the time: 5-7 days"). The CES-D provides a total score, as well as four subscales: negative affect, positive affect, somatic symptoms, and interpersonal problems. A cutoff score of 16 or more is considered "significant" depression.

\section{Catastrophizing}

The Pain Catastrophizing Scale assessed participants' appraisal process of the pain experience. ${ }^{17}$ The scale consists of 13 items for which participants indicated the degree to which they have the thoughts when in pain, ranging from 0 ("not at all") to 4 ("all the time"). The Pain Catastrophizing Scale provides a total score with three subscales (rumination, magnification, and helplessness).

\section{Suicidal ideation}

SI was measured using participants' response to a single item on the Patient Health Questionnaire Nine (PHQ-9) scale. ${ }^{18}$ The PHQ-9 assesses depressive symptoms within the past two weeks. Item 9 assesses SI specifically by asking participants to indicate how often they have been bothered by thoughts that they would be better off dead or of hurting themselves in some way. Participants who reported that they had these types of thoughts "several days," "more than half of the days," or "nearly every day" were considered to have SI; participants who responded "not at all" were considered to have no SI.

\section{Data analyses}

Data analyses were conducted using SPSS version 21 . The sample size of 190 patients provided sufficient power based on regression recommendations $(n=60-80)$. Analysis of variance (ANOVAs) were run across centres for patients' age, 
level of education, time since diagnosis, and $\mathrm{SI}$ to examine potential abnormalities/bias in data collection. Frequencies produced the percentage of cases and controls endorsing SI. Zero-order correlations were conducted for the psychosocial risk factors (depression, catastrophizing) and SI for cases and controls to see if pain associated psychological factors seems to differentiate the prediction of SI and to examine possible age effects on SI. Univariate regressions quantified the associations between psychosocial factors and SI. Followup analyses were performed when SI was significantly predicted by variables with subscales. A final multivariable regression, biopsychosocially conceptualized, determined the relative predictive significance of psychosocial factors on SI, with demographic and medical entered before psychosocial variables.

\section{Results}

As shown in Table 1, the patient sample predominantly consists of middle-aged, White, married females, mostly unemployed, reporting an average length of diagnosis of seven years. The controls are similarly middle-aged, White, married females, and employed. ANOVAs across participating centres ruled out bias in data collection, with no differences reported for $\mathrm{SI}(\mathrm{p}=0.724)$, patient age $(\mathrm{p}=0.391)$, level of education ( $p=0.457)$, or time since diagnosis $(p=0.108)$.

In Fig. 1, 23.2\% of the patient sample reported SI vs. $6 \%$ of controls. The majority of patients endorsed "several days" $(13.2 \%)$ over the past two-weeks when asked about SI, with a minority endorsing "more than half the days" $(6.8 \%)$ or "nearly every day" (3.2\%). Controls that endorsed SI also mainly reported "several days" (5.1\%), with "more than half the days" (0.9\%). No controls endorsed SI "nearly every day."

There are several variables associated with active $\mathrm{SI}$ in the patient sample, the most notable being the psychosocial variables of depression and catastrophizing (i.e., ICSI 0.24; ICPI 0.24; pain 0.25; catastrophizing 0.35; depression $0.64 ; \mathrm{p}<0.01)$. There is no evident correlation pattern where construct redundancy for regression is possible. Further, correlations for the psychosocial risk factors and SI across controls and cases showed for controls, that SI was associated with greater pain $(r=0.31 ; p<0.01)$ and depression $(r=0.59 ; p<0.01)$. Whereas for patients, $S I$ was associated with pain $(r=0.24 ; p<0.01)$, depression $(r=0.64 ; p<0.01)$, and catastrophizing $(r=0.35 ; p<0.01)$. Finally, correlations were also conducted to examine age effects on $\mathrm{Sl}$, showing that patient age was associated with greater length of diagnosis $(r=0.26 ; p<0.01)$, but not with increased $\mathrm{SI}(r=-0.06 ; p=0.41)$ or depression ( $r=-0.09 ; p=0.25$ ).

Univariate regressions were performed to identify variables associated with SI. Table 2 shows that catastrophizing was a predictor of $\mathrm{SI}$, with followup analysis showing

\begin{tabular}{|c|c|c|}
\hline Variable & $\begin{array}{c}\text { IC/BPS } \\
\text { patients }(n=190)\end{array}$ & $\begin{array}{c}\text { Healthy } \\
\text { controls }(n=117)\end{array}$ \\
\hline Age (range; mean \pm SD) & $\begin{array}{c}21-89 \\
49.20 \pm 14.94\end{array}$ & $\begin{array}{c}20-74 \\
47.83 \pm 13.52\end{array}$ \\
\hline $\begin{array}{l}\text { Length of diagnosis } \\
\text { (range; mean } \pm \mathrm{SD} \text { ) }\end{array}$ & $\begin{array}{c}0.50-40 \\
7.10 \pm 6.05\end{array}$ & Not applicable \\
\hline \multicolumn{3}{|l|}{ Ethnicity } \\
\hline White & $89.6 \%$ & $82.1 \%$ \\
\hline Black & $0.5 \%$ & $5.1 \%$ \\
\hline Latino & $1.6 \%$ & $0.9 \%$ \\
\hline American-Indian/Native & $2.6 \%$ & $0.9 \%$ \\
\hline Asian & $5.2 \%$ & $11.1 \%$ \\
\hline \multicolumn{3}{|l|}{ Relationship status } \\
\hline Single & $13.0 \%$ & $19.7 \%$ \\
\hline Married & $59.6 \%$ & $56.4 \%$ \\
\hline Living with partner & $12.4 \%$ & $8.5 \%$ \\
\hline Separated & $2.1 \%$ & $3.4 \%$ \\
\hline Divorced & $8.3 \%$ & $10.3 \%$ \\
\hline Widowed & $4.7 \%$ & $1.7 \%$ \\
\hline \multicolumn{3}{|l|}{ Education } \\
\hline Less than high school & $5.2 \%$ & $2.7 \%$ \\
\hline High school & $16.6 \%$ & $15.8 \%$ \\
\hline Some college & $32.6 \%$ & $28.1 \%$ \\
\hline College degree & $33.7 \%$ & $30.7 \%$ \\
\hline Advanced degree & $11.4 \%$ & $22.8 \%$ \\
\hline \multicolumn{3}{|l|}{ Employment } \\
\hline Homemaker & $11.4 \%$ & $4.3 \%$ \\
\hline Unemployed & $2.6 \%$ & $0 \%$ \\
\hline Retired & $18.1 \%$ & $14.7 \%$ \\
\hline Disabled & $22.8 \%$ & $2.6 \%$ \\
\hline Employed & $43.5 \%$ & $73.2 \%$ \\
\hline Student & $1.0 \%$ & $5.2 \%$ \\
\hline \multicolumn{3}{|l|}{ Measures (mean $\pm S D$ ) } \\
\hline IC symptom index & $12.47( \pm 4.65)$ & $2.38( \pm 2.32)$ \\
\hline IC problem index & $10.96( \pm 3.91)$ & $1.30( \pm 2.36)$ \\
\hline Total pain score & $19.39( \pm 10.61)$ & $3.40( \pm 6.15)$ \\
\hline Catastrophizing total & $21.82( \pm 12.64)$ & $9.89( \pm 9.20)$ \\
\hline Depression total & $19.28( \pm 13.37)$ & $7.33( \pm 8.40)$ \\
\hline
\end{tabular}

the helplessness subscale as the significant predictor of SI. Depression was also a predictor of SI, with negative affect and a lack of positive affect as significant predictors of SI.

The multivariable regression in Table 3 examined patient SI using psychosocial risk factors while controlling for demographic and IC symptoms. Catastrophizing and depression accounted for a significant variance over and above the IC symptoms $\left(\Delta R^{2}=0.32 ; p<0.01\right)$. The final model accounts for approximately $41 \%$ of the variance for patient SI with depression as the lone significant predictor of SI.

\section{Discussion}

The $23 \%$ SI prevalence in this tertiary care sample of IC/ BPS patients is more than double that previously reported in community-dwelling participants with self-reported IC/BPS. ${ }^{8}$ 


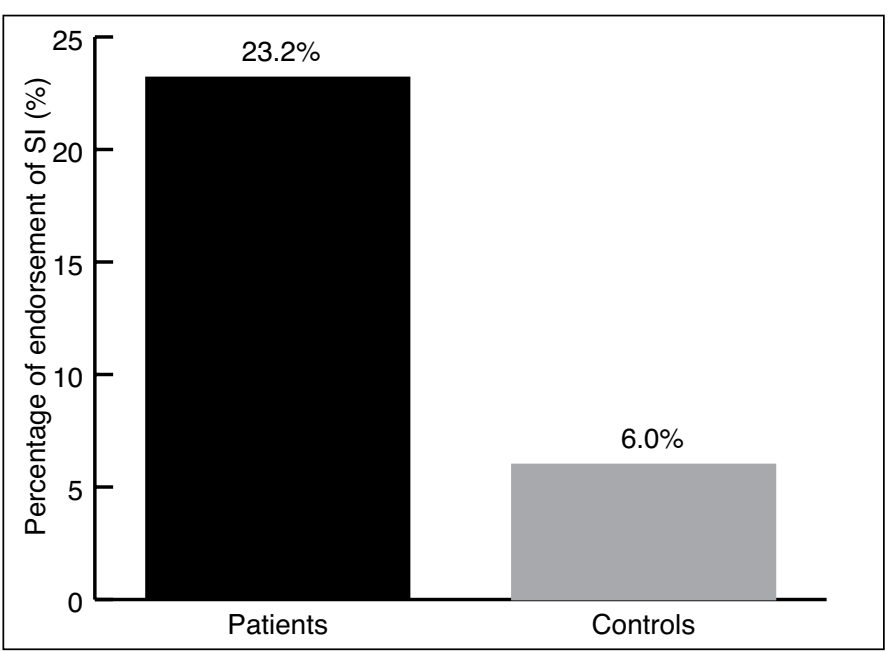

Fig. 1. Prevalence of suicidal ideation (SI) in patients with interstitial cystitis/ bladder pain syndrome and controls.

An elevated endorsement of SI was hypothesized in tertiary care IC/BPS patients compared to primary care cases, which is also supported by existing research for $\mathrm{SI}$ in other samples of abdominal pelvic pain (e.g., 38\% of irritable bowel syndrome cases vs. $4 \%$ primary care patients). ${ }^{11}$

Correlations of age on SI showed that older patients had a longer length of time since diagnosis, but age was not associated with increased reporting of SI or with level of depression. This is important because it may be speculated that older patients can expect to report longer disease histories with more years of significant symptoms and, thus, perhaps a higher risk for depression or SI. Further, correlations were also used to examine the associations of SI with the psychosocial risk factors of depression and catastrophizing across cases and controls. The results showed that for controls, greater SI was associated with greater pain and depression. Whereas, for cases, greater SI was associated with more pain, depression, and pain catastrophizing. This implies that for patients only, the pain associated psychological risk factors differentiate SI prediction.

The present findings add new considerations to previous research ${ }^{8}$ showing that catastrophizing and depression are uniquely important in predicting suicidality. In particular, the current results show that catastrophizing, as well as depression, predict SI over and above pain and IC/BPS symptoms. This new finding for catastrophizing as a predictor of $\mathrm{SI}$ in IC/BPS is congruent with its role as a key psychological predictor of negative pain-related outcomes, ${ }^{19-21}$ its associations with depression and poor mental quality of life, and its prediction of suicidality in patients with chronic pain. ${ }^{13}$ In particular, helplessness was identified as a key catastrophizing domain for SI, which has also previously been associated with greater pain, diminished mental quality of life, and sexual functioning in IC/BPS. ${ }^{19,21}$

Depression was strongly associated with $\mathrm{SI}$ and was elevated in this study vs. controls, as well as those reported by
Table 2. Univariate regression analysis with catastrophizing and depression predicting suicidal ideation

\begin{tabular}{lccccc}
\hline Model 1 & $\mathbf{R}^{\mathbf{2}}$ & $\begin{array}{c}\text { Adjusted } \\
\mathbf{R}^{2}\end{array}$ & $\mathbf{F}$ & $\beta$ & $\mathbf{t}$ \\
\hline Catastrophizing & 0.12 & 0.12 & $25.35^{* *}$ & & \\
$\quad$ Rumination & & & & -0.23 & -1.83 \\
Helplessness & & & & $0.51^{* *}$ & 4.14 \\
Magnification & & & & 0.10 & 0.98 \\
& & & & & \\
Depression & 0.41 & 0.41 & $121.26^{* *}$ & & \\
$\quad$ Negative affect & & & & $0.39^{* *}$ & 4.36 \\
Positive affect* & & & & $0.33^{* *}$ & 4.30 \\
$\quad$ Somatic symptoms & & & & 0.01 & 0.08 \\
Interpersonal & & & & 0.02 & 0.32 \\
$\quad$ problems & & & & & \\
\hline *Reverse scored;** $p<0.01$. & & & & \\
\hline
\end{tabular}

Hepner et al. ${ }^{8}$ The depression scores in this study are suggestive of significant depressive symptoms in these patients, ${ }^{16}$ and it is well known that depression is one of the strongest risk factors for suicide. ${ }^{22}$ In this study, depression was a univariate predictor of $\mathrm{SI}$ and was also the strongest multivariable predictor of SI over patient pain and symptoms. Patients reporting concomitant pain and depression have a reduced likelihood of recovery and longer symptom duration. ${ }^{23}$ With depression estimates in IC/BPS varying from $5 \%$ to greater than $50 \%$ of samples, ${ }^{12,24,25}$ and pain as a cardinal symptom, ${ }^{2}$ identification of $\mathrm{SI}$ in tertiary care is recommended.

Although depression in this study has a stronger association with SI than catastrophizing, depression and catastrophizing likely manifest in tandem. Moreover, catastrophizing is a cognitive stress-vulnerability factor related to depression in chronic pain patients, ${ }^{26}$ and over time, catastrophizing can lead to depression. ${ }^{26}$ Thus, urologists treating women with IC/BPS should recognize patient helplessness in regards to pain. Patients may report excessive worry that the pain will never improve or that they are completely overwhelmed and feel there is nothing they can do. Such reports by patients can be followed up with discussions aimed at identifying and managing these stressful thinking patterns. ${ }^{27}$ Further, given the present findings, it may be prudent to make oneself familiar with recommendations for treating suicide risk. ${ }^{28}$ Such treatment plans have been more successful than no-suicide contracts in preventing suicidal behaviours. ${ }^{29}$ What is clear, given the present data, is that depression/suicide screening should be a standard domain in patient evaluations. Indeed, consisting of results from well-designed studies in representative populations, the U.S. Preventive Service Task Force (USPSTF) has recently issued a recommendation screening for depression in the general adult population. ${ }^{30}$ One recommended screening measure would be the PHQ-9. ${ }^{18}$ This measure is brief and useful in clinical practice because it is completed by patients in minutes and can be rapidly scored. The PHQ-9 can also be administered repeatedly, which can be used to record improvement or worsening of depres- 


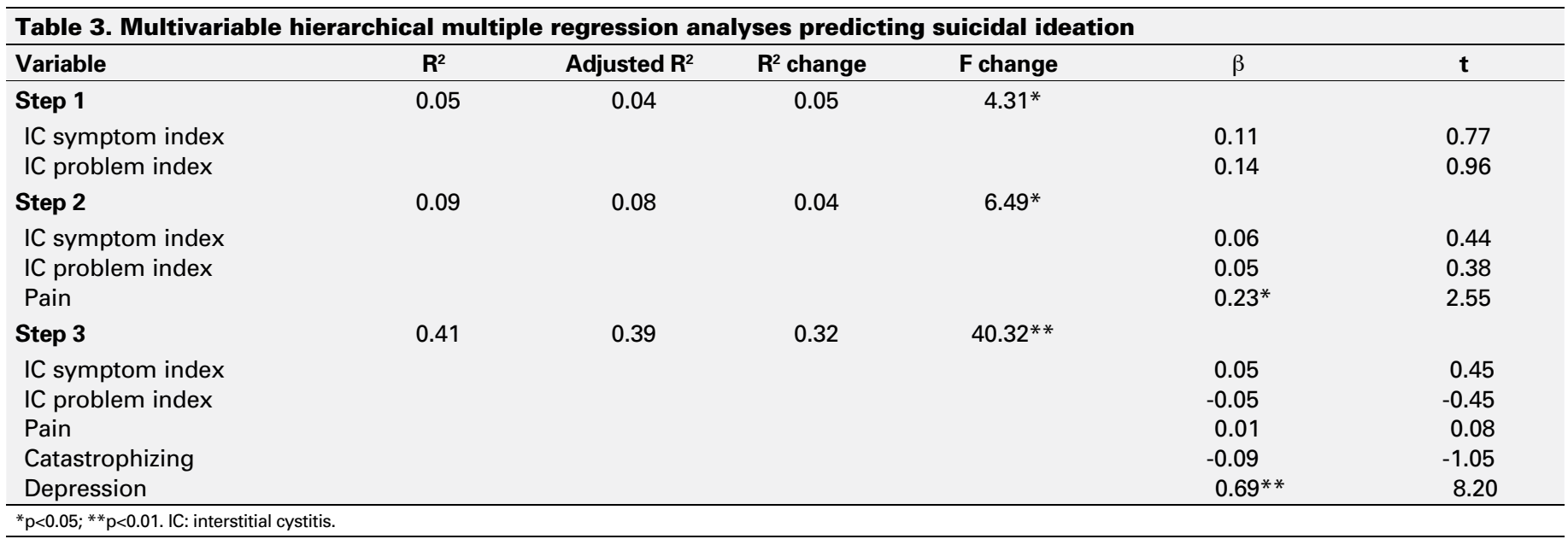

sion or $\mathrm{SI}$ in response to interventions. When $\mathrm{SI}$ is present, clinicians should also follow up by requesting details on the presence or absence of specific suicide plans, including any steps taken towards a plan, like preparations for death. It is always important to obtain as much detail as possible about the timing, intent, method, and consequences of such behaviours. As well, clinicians should be wary and query SI again if there have been sudden changes in clinical status, which may include worsening or precipitous and unexpected improvements in symptoms. The American Psychiatric Association has published best practice guidelines on the management of identified suicidal risk, ${ }^{31}$ and it is suggested that referral to a psychiatrist or psychologist is recommended as part of a multidisciplinary treatment plan.

Limitations of this study include its cross-sectional design and primarily correlation-based statistics, which mean that causality or direction cannot be established on which came first, pain or SI. SI is an important predictor of suicidality, but does not fully predict suicide mortality. Thus, the current results should not be used as a lone indicator of suicide mortality. This study is also limited in its ability to understand SI using a one-item response from the PHQ-9 questionnaire. While this index has been used in previous research, ${ }^{8}$ future urology research should use standardized norm-based SI assessments examining past history of SI, motivation, and future suicidality risk. It must also be stated that the generalizability of the study is limited to female patients receiving treatment in tertiary care urology centres and thus, there could be some concern over the severity of patients' symptoms. A particular issue of generalizability in this study is that men also suffer from IC/BPS, but are not included in the present sample. As suggested by Naliboff et $\mathrm{al}^{32}$ psychosocial and somatic symptom comorbidities are important for treatment planning in chronic pelvic pain syndromes. It is important to better characterize these variables in both men and women suffering from IC/BPS. The process of consecutive referrals in this study provided equal access for all attending patients on study days across clinics for approximately four months. Although this SI data in tertiary care IC/BPS centres augments the literature and does create a reflection of tertiary care practice, the data is based on retrospective self-report and is subject to some recall bias and willingness to admit suicide-related behaviours. Thus, replications of these findings are required to establish a more true prevalence rate. However, it should also be considered that the use of the self-administered format for suicide questions, where the person reads and responds privately, likely improved the response rate. Finally, this study used a single point prevalence estimate for IC/BPS SI, not permitting examination of any changes in SI over time. A prospective design is needed to further understand changes in SI and risk factors, as well as to study potential important covariates, such as the active use of antidepressants or neuroleptic medications.

Further research with patients suffering from IC/BPS could also investigate the specific impact of chronic vs. acute pain flares and the association to SI. Other areas of interest may include the impact that different types of pain (e.g., medically unexplained pain) can have in IC/BPS and SI, and a deeper examination of other risk factors shown in $\mathrm{SI}$ in other chronic pain patients. ${ }^{33,34}$

\section{Conclusion}

This study indicates that tertiary care patients with IC/BPS have an alarming rate of SI. Depression, catastrophizing that is characterized by helplessness about managing pain, and pain are all significantly associated with higher SI. The results highlight the importance of identifying and treating comorbid mental disorders common in patients with IC/ BPS. The results also should impress upon clinicians the need for identification of potential suicide risk in tertiary IC/BPS patients, especially since SI may not be forecast by traditionally used IC/BPS symptom measures in isolation. 
Tripp et al.

Competing interests: Dr. Nickel has been an advisor for Astellas, Auxillium, Eli Lilly, Farr Labs, Ferring, GSK, Pfizer, Taris Biomedical, Tribute Pharmaceuticals, and Trillium Therapeutics; a speaker for Astellas and Eli Lilly; and has participated in clinical trials for Eli Lilly, GSK, Johnson \& Johnson, Pfizer, and Taris Biomedical. Dr. Pontari has been an advisor for Eli Lilly and Tribute Pharmaceuticals. Dr. Moldwin has been an advisor for Allergan, Aquinox, and Urigen; has received honoraria from Allergan; and has participated in clinical trials for Allergan, Afferon, and Urigen. Dr. Carr has been an advisor and speaker for Allergan, Astellas, Ferring, and Pfizer; and has participated in clinical trials for Allergan, Astellas, Aquinox, and Cook MyoSite. Dr. Yang has been an advisor for Tribute Pharmaceuticals. Dr. Nordling has been an advisor for Allergan and Aquinox and has received honoraria from Astellas. The remaining authors report no competing personal or financial interests.

This paper has been peer-reviewed.

\section{References}

1. Rosamilia A. Painful bladder syndrome/interstitial cystitis. Best Pract Res Clin Obstet Gynaecol 2005;19:843-59. http://dx.doi.org/10.1016/i.bpobgyn.2005.08.004

2. Tripp DA, Nickel JC, Wong J, et al. Mapping of pain phenotypes in female patients with bladder pain syndrome/interstitial cystitis and controls. Eur Urol 2012;62:1188-94. http://dx.doi.org/10.1016/i. eururo.2012.05.023

3. Hanno PM, Burks DA, Clemens JQ, et al. AUA guideline for the diagnosis and treatment of interstitial cystitis/ bladder pain syndrome. J Urol 2011;185:2162-70. http://dx.doi.org/10.1016/i.juro.2011.03.064

4. Stenager $\mathrm{E}$, Christiansen $\mathrm{E}$, Handberg $\mathrm{G}$, et al. Suicide attempts in chronic pain patients. A register-based study. Scand J Pain 2014;5:4-7. http://dx.doi.org/10.1016/i.spain.2013.09.001

5. Hitchcock LS, Ferrell BR, McCaffrey M. The experience of chronic non-malignant pain. J Pain Symptom Manage 1994;9:312-8. http://dx.doi.org/10.1016/0885-3924(94)90190-2

6. Magni $G$, Rigatti-Luchini S, Fracca F, et al. Suicidality in chronic abdominal pain: An analysis of the Hispanic Health and Nutrition Examination Survey (HHANES). Pain 1998;76:137. http://dx.doi.org/10.1016/ S0304-3959(98)00035-9

7. Fishbain DA. Current research on chronic pain and suicide. Am J Public Health 1996;86:1320-1. http://dx.doi.org/10.2105/AJPH.86.9.1320

8. Hepner KA, Watkins KE, Watkins MN, et al. Suicidal ideation among patients with bladder pain syndrome/ interstitial cystitis. Urology 2012;80:280-5. http://dx.doi.org/10.1016/i.urology.2011.12.053

9. Berry SH, Bogard LM, Pham C, et al. Development, validation and testing of an epidemiological case definition of interstitial cystitis/painful bladder syndrome. J Urol 2010;183:1848-52. http://dx.doi. org/10.1016/i.juro.2009.12.103

10. Kessler RC, Borges $G$, Walters EE. Prevalence of and risk factors for lifetime suicide attempts in the National Comorbidity Survey. Arch Gen Psychiatry 1999:56:617-26. http://dx.doi.org/10.1001/ archpsyc.56.7.617

11. Miller V, Hopkins L, Whorwell PJ. Suicidal ideation in patients with irritable bowel syndrome. Clin Gastroenterol Hepatol 2004;2:1064-8. http://dx.doi.org/10.1016/S1542-3565(04)00545-2

12. Clemens IQ, Brown SO, Calhoun EA. Mental health diagnoses in patients with interstitial cystitis/painful bladder syndrome and chronic prostatitis/chronic pelvic pain syndrome: A case/control study. J Urol 2008;180:1378-82. http://dx.doi.org/10.1016/i.juro.2008.06.032

13. Edwards RR, Smith MT, Kudel I, et al. Pain-related catastrophizing as a risk factor for suicidal ideation in chronic pain. Pain 2006;126:272-9. http://dx.doi.org/10.1016/i.pain.2006.07.004

14. O'Leary MP, Sant GR, Fowler FJ Jr, et al. The interstitial cystitis symptom index and problem index. Urology 1997;49:58-63. http://dx.doi.org/10.1016/S0090-4295(99)80333-1

15. Melzack R. The short-form McGill Pain Questionnaire. Pain 1987;30:191-7. http://dx.doi. org/10.1016/0304-3959(87)91074-8
16. Radloff L. The CES-D scale: A self-report depression scale for research in the general population. Appl Psychol Meas 1977;1:385-401. http://dx.doi.org/10.1177/014662167700100306

17. Sullivan MJ, Bishop SR, Pivik J. The pain catastrophizing scale: Development and validation. Psychol Assess 1995;7:524-32. http://dx.doi.org/10.1037/1040-3590.7.4.524

18. Kroenke K, Spitzer RL, Williams JBW. The PHQ-9: Validity of a brief depression severity measure. J Gen Intern Med 2001;16:606-13. http://dx.doi.org/10.1046/j.1525-1497.2001.016009606.x

19. Nickel JC, Tripp DA, Teal $V$, et al. Sexual function is a determinant of poor quality of life for women with treatment refractory interstitial cystitis. J Urol 2007;177:1832-6. http://dx.doi.org/10.1016/i. juro.2007.01.060

20. Tripp DA, Nickel JC, Wang Y, et al. Catastrophizing and pain-contingent rest predict patient adjustment in men with chronic prostatitis/chronic pelvic pain syndrome. J Pain 2006;7:697-708. http://dx.doi. org/10.1016/i.jpain.2006.03.006

21. Tripp DA, Nickel JC, FitzGerald MP, et al. Sexual functioning, catastrophizing, depression, and pain as predictors of quality of life in women with interstitial cystitis/painful bladder syndrome. Urology 2009;73:987-92. http://dx.doi.org/10.1016/j.urology.2008.11.049

22. Cheng ATA, Chen THH, Chen $\mathrm{CC}$, et al. Psychosocial and psychiatric risk factors for suicide. Br J Psych 2000;177.

23. Bair MJ, Robinson RL, Katon W, et al. Depression and pain comorbidity: A literature review. Arch Intern Med 2003;163:2433-45. http://dx.doi.org/10.1001/archinte.163.20.2433

24. Goldstein $H B$, Safaeian $P$, Garrod $K$, et al. Depression, abuse and its relationship to interstitial cystitis. Int Urogynecol J Pelvic Floor Dysfunct 2008; 19:1683-6. http://dx.doi.org/10.1007/s00192-008-0712-x

25. Rothrock NE, Lutgendorf SK, Hoffman A, et al. Depressive symptoms and quality of life in patients with interstitial cyssitis. J Urol 2002;167:1763-7. http://dx.doi.org/10.1016/S0022-5347(05)65195-6

26. Lee EJ, Wu MY, Lee GK, et al. Catastrophizing as a cognitive vulnerability factor related to depression in workers' compensation patients with chronic musculoskeletal pain. J Clin Psychol Med Settings 2008;15:182-92. http://dx.doi.org/10.1007/s10880-008-9118-7

27. Tripp DA, Nickel JC. The psychology of urological chronic pelvic pain: A primer for urologists who want to know how to better manage chronic prostatitis and interstitial cystitis. AUA Update Series, Lesson 40, Volume 302011

28. Bryan CJ, Corso KA, Neal-Walden TA, et al. Managing suicide risk in primary care: Practice recommendations for behavioural health consultants. Prof Psychol: Res Practice 2009;40:148-55. http://dx.doi. org/10.1037/00011141

29. Rudd MD, Mandrusiak M, Joiner TE Jr. The case against no-suicide contracts: The commitment to treatment statement as a practice alternative. J Clin Psychol 2006;62:243-51. http://dx.doi.org/10.1002/ ¡ilp.20227

30. Siu AL, Bibbins-Domingo K, Grossman DC, et al. Screening for depression in adults: US Preventive Services Task Force recommendation statement. JAMA 2016;315:380-7. http://dx.doi.org/10.1001/ jama.2015.18392

31. Jacobs DG, Baldessarini RJ, Conwell Y, et al. Practice guideline for the assessment and treatment of patients with suicidal behaviours. American Psychiatric Association, 2003.

32. Naliboff BD, Stephens AJ, Afari N, et al. Widespread psychosocial difficulties in men and women with urologic chronic pelvic pain syndromes: Case-control findings from the Multidisciplinary Approach to the Study of Chronic Pelvic Pain Research Network. Urology 2015;85:1319-27. http://dx.doi.org/10.1016/i. urology.2015.02.047

33. Calati $\mathrm{R}$, Bakhiyi $\mathrm{CL}$, Artero $\mathrm{S}$, et al. The impact of physical pain on suicidal thoughts and behaviours: Meta-analyses. J Psychiatr Res 2015;71:16-32. http://dx.doi.org/10.1016/i.jpsychires.2015.09.004

34. Koenig J, Oelkers-Ax R, Parzer P, et al. The association of self-injurious behaviour and suicide attempts with recurrent idiopathic pain in adolescents: Evidence from a population-based study. Child Adolesc Psychiatry Ment Health 2015;9:32. http://dx.doi.org/10.1186/s13034-015-0069-0

Correspondence: Dr. Dean A. Tripp, Departments of Psychology, Anesthesiology \& Urology, Queen's University, Kingston, ON, Canada; dean.tripp@queensu.ca 